\title{
TREINAMENTO COMBINADO DE SPRINTS E AGACHAMENTO MELHORA A MODULAÇÃO AUTONÔMICA CARDÍACA DE JOVENS SAUDÁVEIS
}

Natanael Pereira Batista, Aryane Flauzino Machado, Hygor Ferreira da Silva, Caio Russo Dutra Rodrigues, Flavia Alves de Carvalho, Larissa Rodrigues Souto, Altair Custódio Junior, Carlos Marcelo Pastre.

Universidade Estadual Paulista "Júlio de Mesquita Filho" - FCT UNESP, Departamento de Fisioterapia, Presidente Prudente/SP. E-mail: natan.pbatista@gmail.com

FAPESP - Fundação de Amparo à Pesquisa do Estado de São Paulo (n²015/25220-9)

\section{RESUMO}

Estudos demonstram efeitos benéficos do treinamento físico sobre a modulação autonômica cardíaca. 0 treinamento combinado, caracterizado por diferentes estímulos em uma mesma sessão, tem sido bem utilizado devido seus benefícios sobre a performance. O objetivo do estudo foi avaliar a modulação autonômica cardíaca por meio da variabilidade da frequência cardíaca (VFC) de jovens após um treinamento combinado. Trinta e oito jovens saudáveis realizaram 6 semanas de treinamento combinado de sprints e agachamento. A VFC foi avaliada em repouso no início e ao final do treinamento completo. Os índices RMSSD (root mean square of sucessive differences / raiz quadrada média das diferenças sucessivas de intervalos RR) e SDNN (standard deviation of $R R$ intervals / desvio padrão dos intervalos RR) apresentaram aumento estatisticamente significante após o treinamento $(p=0,016 ; E S=0,410 ; p \leq 0,001$; $\mathrm{ES}=0,647$, respectivamente). Portanto, conclui-se que o treinamento combinado de 6 semanas foi eficaz para melhora da modulação autonômica cardíaca, avaliada por meio da VFC.

Palavras-chave: exercício, sistema nervoso autônomo, recuperação da função fisiológica.

\section{COMBINED TRAINING OF SPRINTS AND SQUATS IMPROVES CARDIAC AUTONOMIC MODULATION OF HEALTHY YOUNG MEN}

\begin{abstract}
Studies demonstrate beneficial effects of physical training on cardiac autonomic modulation. Combined training, characterized by different stimulus in the same session, has been widely used because of its performance benefits. The aim of the study was to evaluate the cardiac autonomic modulation through heart rate variability (HRV) of young men after a combined training. Thirty eight healthy young men performed 6 weeks of combined training of sprints and squats. HRV was measured during rest at the beginning and the end of the full training. The indexes RMSSD (root-mean square of successive differences) and SDNN (standard deviation of normal RR intervals) showed significant increase after the training $(p=0.016 ; E S=0.410 ; p \leq 0.001 ; E S=0.647$, respectively). Therefore, it is concluded that the combined training during 6 weeks was effective to improve cardiac autonomic modulation, evaluated through HRV.
\end{abstract}

Keywords: exercise, autonomic nervous system, recovery of function.

\section{INTRODUÇÃO}

O treinamento físico é parte fundamental do âmbito esportivo e pode ser definido como um processo repetitivo e sistemático composto de exercícios progressivos que visam o aperfeiçoamento do desempenho ${ }^{1}$. A dinâmica dos programas de treinamento pode ser modificada e adequada de acordo com os objetivos a serem atingidos ${ }^{2}$. Um dos exemplos é Colloq Vitae 2018 set-dez; 10(3): 60-65. DOI: 10.5747/cv.2018.v10.n3.v244 ISSN 1984-6436/C 2018 - Publicado pela Universidade do Oeste Paulista. Artigo Open Access sob uma licença CC BY-NC-ND (http://creativecommons.org/licenses/by-nc-nd/4.0/). a combinação de diferentes estímulos em uma mesma sessão, denominado treinamento combinado, e que tem sido utilizado comumente no cenário esportivo. Estudos apontam que essa combinação é capaz de produzir melhores respostas adaptativas, e consequentemente otimizar ganhos de desempenho em volumes reduzidos de treinamentos ${ }^{2-4}$. Marques et $a l^{4}$ aplicaram um programa de treinamento 
combinado de sprints e agachamento, e o compararam com ambos os estímulos isoladamente, evidenciando que sua combinação na mesma sessão foi capaz de otimizar o ganho de desempenho em sprints e força. Além disso, comparado a treinamentos nos quais os estímulos são propostos em sessões ou dias diferentes, bons resultados têm sido evidenciados a favor da realização dos estímulos na mesma sessão ${ }^{5}$.

Volume e intensidade são fatores que compõe a construção e elaboração do treinamento e seu controle é fundamental para evitar alterações fisiológicas indesejáveis, bem como otimizar a adaptação ao treinamento ${ }^{6,7}$. Como forma de monitoramento e controle das cargas de treino, uma estratégia que tem sido utilizada é análise da modulação autonômica cardíaca por meio da variabilidade da frequência cardíaca $(\mathrm{VFC})^{8}$. De maneira geral, essa análise descreve oscilações entre batimentos cardíacos consecutivos e estes por sua vez estão associados a atuação do sistema nervoso autônomo (SNA) sobre o nódulo sinusal e o controle da modulação autonômica cardíaca. Sendo assim, é uma ferramenta de medida não invasiva que pode identificar fenômenos relacionados ao SNA em indivíduos saudáveis, atletas e portadores de doença ${ }^{9-11}$.

As variações da frequência cardíaca podem ser avaliadas de algumas formas. A mais simples e mais utilizada se dá pelo domínio do tempo da VFC, que representa o tempo entre os batimentos cardíacos. No sinal eletrocardiográfico cada complexo QRS é detectado e a partir dos intervalos entre cada complexo (intervalos RR) é possível determinar as atuações do sistema nervoso autônomo em diversas variáveis. A revisão sistemática de Bellenger et al. $^{12}$ observaram que ganhos de desempenho após um programa de treinamento físico estão relacionados com aumento da atividade vagal, avaliada por meio da VFC, destacando a modulação parassimpática cardíaca como uma adaptação positiva ao treinamento ${ }^{8,12}$.

Tendo em vista as características do treinamento combinado, parece pertinente entender se diferentes estímulos fornecidos em uma mesma sessão de treinamento geram adaptações na modulação autonômica cardíaca. Sendo assim, o objetivo do presente estudo foi avaliar a variabilidade da frequência cardíaca de jovens saudáveis após seis semanas de treinamento combinado de sprints $\mathrm{e}$ agachamento.

\section{METODOLOGIA}

Amostra

O estudo foi composto de 38 jovens aparentemente saudáveis. Para serem incluídos os participantes deveriam ter idade entre 18 e 30 anos, reportar ausência de lesão musculoesquelética nos últimos seis meses, doenças cardiovasculares, uso de drogas antiinflamatórias e analgésicas, bem como a prática de qualquer exercício físico não proposto pelo estudo. A presente pesquisa foi previamente aprovada pelo Comitê de Ética em Pesquisa da Faculdade de Ciências e Tecnologia da Universidade Estadual Paulista "Júlio de Mesquita Filho" - FCT/UNESP (parecer no 2016/1.389.046). Os participantes foram devidamente informados sobre os objetivos e procedimentos deste estudo e, após concordarem, assinaram um Termo de Consentimento Livre e Esclarecido passando a fazer parte efetivamente do mesmo.

\section{Delineamento do estudo}

O presente estudo é um ensaio clínico. A coleta de dados foi realizada em um centro de atendimento de Fisioterapia e reabilitação na Faculdade de Ciências e Tecnologia - FCT/UNESP, respeitando o horário das $17 \mathrm{~h} 30$ às $22 \mathrm{~h} 00$. Todos os protocolos foram realizados conforme experimentos já realizados de mesma natureza em um laboratório sob condições padronizadas (temperatura: $22 \pm 1^{\circ} \mathrm{C}$, umidade relativa: 40 $60 \%)^{13}$.

Inicialmente foram coletados dados antropométricos para caracterização da amostra, sobre idade, peso, altura e IMC. A análise da estatura dos participantes foi realizada por meio de um estadiômetro (Sanny - American Medical do Brasil, São Paulo, Brasil) e a massa corpórea foi avaliada por meio de uma balança digital (Tanita BC554, Iron Man/Inner Scaner - Tanita, Illinois, EUA). Todos os participantes também realizaram uma avaliação da variabilidade da frequência cardíaca, e para tal foram orientados a não realizar exercícios físicos rigorosos por $24 \mathrm{~h}$, não consumir bebidas alcóolicas e/ou estimulantes tais como café, chá e refrigerantes a base de cola, realizar uma refeição leve pelo menos duas horas antes dos procedimentos, bem como vestir roupas e calçados confortáveis no dia da avaliação. 


\section{Protocolo de treinamento combinado}

Trata-se de um programa de treinamento descrito por Marques et al. ${ }^{4}$. Tal programa é composto por treinos de sprints e agachamentos realizados na mesma sessão, duas vezes por semana durante um período de 6 semanas. As sessões de treino se iniciam por aquecimento específico de sprint seguido do treinamento de sprints. Todos os participantes realizaram as mesmas distancias, sendo essas progredidas de acordo com a Tabela 1. Logo após, os participantes realizaram um intervalo de descanso de 10 min para em seguida iniciar o aquecimento e treino de agachamento na barra guiada. As cargas utilizadas foram baseadas em percentuais de carga máxima movida em $1 \mathrm{~m} / \mathrm{s}$ em exercício de agachamento na barra guiada, conforme descrito por Machado et al. ${ }^{14}$.

Tabela 1. Programa de treinamento combinado de 6 semanas.

\begin{tabular}{|c|c|c|c|c|c|c|c|c|c|}
\hline \multirow{3}{*}{ Sem. } & \multirow{3}{*}{ Sessão } & \multicolumn{3}{|c|}{ Treino de Sprint } & & \multicolumn{4}{|c|}{ Treino de agachamento } \\
\hline & & Aquect & Séries $x$ & Descanso & & Aquect+ & Séries $x$ & Intensidade & Descanso \\
\hline & & & Repetições & & \multirow{9}{*}{ 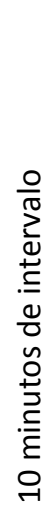 } & & Repetições & $\%$ & \\
\hline \multirow{2}{*}{1} & 1 & $3 \times 20 m$ & $2 \times 15 m$ & $120 s$ & & $1 \times 8+1 \times 8$ & $1 \times 8$ & 80 & $120 s$ \\
\hline & 2 & $3 \times 20 m$ & $2 \times 15 \mathrm{~m}$ & $120 s$ & & $1 \times 8+1 \times 8$ & $2 \times 8$ & 80 & $120 s$ \\
\hline \multirow{2}{*}{2} & 3 & $3 \times 25 \mathrm{~m}$ & $2 \times 20 m$ & $120 s$ & & $1 \times 8+1 \times 8$ & $1 \times 8$ & 85 & $120 s$ \\
\hline & 4 & $3 \times 25 \mathrm{~m}$ & $2 \times 20 m$ & $120 s$ & & $1 \times 8+1 \times 8$ & $2 \times 6$ & 85 & $120 \mathrm{~s}$ \\
\hline \multirow{2}{*}{3} & 5 & $3 \times 30 m$ & $2 \times 25 \mathrm{~m}$ & $150 \mathrm{~s}$ & & $1 \times 8+1 \times 8$ & $1 \times 6$ & 90 & $120 s$ \\
\hline & 6 & $3 \times 30 m$ & $2 \times 25 m$ & $150 s$ & & $1 \times 8+1 \times 8$ & $2 \times 8$ & 90 & $120 s$ \\
\hline \multirow{2}{*}{4} & 7 & $3 \times 35 \mathrm{~m}$ & $2 \times 30 m$ & $180 \mathrm{~s}$ & & $1 \times 8+1 \times 6$ & $1 \times 6$ & 95 & $180 \mathrm{~s}$ \\
\hline & 8 & $3 \times 35 \mathrm{~m}$ & $2 \times 30 m$ & $180 \mathrm{~s}$ & & $1 \times 8+1 \times 6$ & $2 \times 5$ & 95 & $180 \mathrm{~s}$ \\
\hline \multirow{2}{*}{5} & 9 & $3 \times 25 \mathrm{~m}$ & $2 \times 20 m$ & $150 s$ & & $1 \times 8+1 \times 6$ & $2 \times 6$ & 100 & $180 \mathrm{~s}$ \\
\hline & 10 & $3 \times 25 \mathrm{~m}$ & $2 \times 20 m$ & $150 \mathrm{~s}$ & & $1 \times 8+1 \times 6$ & $2 \times 5$ & 100 & $180 \mathrm{~s}$ \\
\hline \multirow{2}{*}{6} & 11 & $3 \times 30 m$ & $1 \times 25 \mathrm{~m}$ & $180 \mathrm{~s}$ & & $1 \times 8+1 \times 6$ & $2 \times 6$ & 90 & $120 \mathrm{~s}$ \\
\hline & 12 & $3 \times 30 \mathrm{~m}$ & $1 \times 25 \mathrm{~m}$ & $180 s$ & & $1 \times 8+1 \times 6$ & $1 \times 8$ & 80 & $120 \mathrm{~s}$ \\
\hline
\end{tabular}

Aquect - Aquecimento a $80 \%$ da velocidade máxima segundo a percepção individual do participante.

Aquect† - Aquecimento com movimento de agachamento lento e controlado.

\section{Análise da variabilidade da frequência cardíaca (VFC)}

A captação da VFC aconteceu por 30 minutos, sendo 10 minutos iniciais de descanso, e os 20 minutos subsequentes considerados para análise. Este procedimento foi realizado da mesma maneira e sob as mesmas condições para captação da VFC basal e final (após o período de seis semanas do treinamento combinado). A análise foi realizada a partir da série de intervalos RR captada pelo cardiofrequencímetro (Polar Electro Oy - modelo V800) e analisados nos domínios do tempo. Para esta análise, a série temporal de intervalos $R R$ foi inicialmente submetida a uma filtragem digital pelo software Kubios HRV - versão 2.0 complementada por uma filtragem manual, para eliminação de batimentos ectópicos prematuros e artefatos, e somente séries com mais de $95 \%$ de batimentos sinusais foram incluídas no estudo.

A série de intervalos RR foi analisada antes e depois das seis semanas de treinamento com 1000 intervalos RR cada análise. Foram avaliados os índices da VFC no domínio do tempo: rMSSD (root mean square of sucessive differences / raiz quadrada da média do quadrado das diferenças entre intervalos RR) e SDNN (standard deviantion of normal RR intervals / desvio padrão de todos os intervalos RR normais). O índice RMSSD representa a modulação parassimpática, enquanto o SDNN é um índice que apresenta correspondência com a potência total do espectro de frequência, ou seja, a variabilidade global, refletindo, portanto, a participação de todos os componentes rítmicos responsáveis pela variabilidade ${ }^{15}$. Foi realizado a 
comparação da média entre os intervalos RR (Mean $R R$ ) do grupo nos momentos pré e póstreinamento para caracterização da amostra, e os índices do domínio do tempo da VFC analisados foram RMSSD e SNDD.

\section{Análise estatística}

Os dados foram analisados utilizando o teste t pareado para comparação das médias dos índices de VFC pré e pós treinamento, e a partir disso foi obtido os valores de tamanho de efeito (Eta-squared), adotado como referência pequeno $(0,01)$, médio $(0,06)$ e grande $(0,14)^{16}$. Toda a análise estatística assumiu o nível de significância de $5 \%$, e valores de média e desvio padrão foram reportados. Foi utilizado o pacote estatístico IBM SPSS versão 20.0.

\section{RESULTADOS}

A Tabela 2 apresenta a caracterização da amostra do estudo.

Foi observado diferença estatisticamente significante entre os momentos pré e póstreinamento para a média entre os intervalos $R R$ (mean RR) e para os índices RMSSD e SDNN, bem como um tamanho de efeito (effect size - ES) considerado grande (Tabela 3).

Tabela 2. Média e desvio padrão das variáveis antropométricas.

\begin{tabular}{lc}
\hline Idade (anos) & $22,8 \pm 3,7$ \\
Altura (cm) & $176,8 \pm 6,1$ \\
Massa corporal (Kg) & $75,3 \pm 12,1$ \\
Índice de massa corporal $\left(\mathrm{Kg} / \mathrm{m}^{2}\right)$ & $24,0 \pm 3,0$ \\
\hline
\end{tabular}

Tabela 3. Valores dos índices da variabilidade da frequência cardíaca (basal e após 6 semanas de treinamento combinado)

\begin{tabular}{cccc} 
& Basal & Após 6 semanas & $\boldsymbol{p}$ \\
\cline { 2 - 4 } MEAN RR & $123,29 \pm 20,00$ & $144,96 \pm 23,51$ & $0,003(\mathrm{ES}=0,443)$ \\
RMSSD & $49,21 \pm 21,28$ & $61,34 \pm 34,01$ & $0,016(\mathrm{ES}=0,410)$ \\
\multirow{2}{*}{ SDNN } & $64,76 \pm 17,49$ & $83,15 \pm 29,43$ & $0,000(\mathrm{ES}=0,647)$
\end{tabular}

Mean RR: Média entre os intervalos RR (expresso em ms);

RMSSD: Root mean square of sucessive differences - raiz quadrada da média do quadrado das diferenças entre intervalos RR (expresso em ms):

SDNN: Standard deviation of normal RR intervals - desvio padrão de todos os intervalos RR normais (expresso em ms); ES: Effect Size - Tamanho de efeito.

\section{DISCUSSÃO}

Este estudo objetivou analisar os efeitos de um treinamento físico combinado de sprints e agachamento sobre a modulação autonômica cardíaca. Foi observado aumento estatisticamente significante do momento póstreinamento em relação ao momento pré, em ambos os índices analisados (RMSSD e SDNN). Os resultados confirmaram nossa hipótese inicial de que o treinamento combinado promoveria melhora nos índices do domínio do tempo da VFC em jovens saudáveis.

Treinamentos físicos periodizados apresentam importantes atuações na melhora de índices vagais da VFC, porém poucos estudos reportam esses efeitos a partir de um treinamento combinado. $O$ presente estudo mostrou que um treinamento combinado de sprints e agachamento, ou seja, estímulos de velocidade e força, durante seis semanas foi capaz de melhorar a atuação parassimpática no repouso. Ainda que com populações diferentes, Amano et al. $^{17}$ aplicaram um programa de treinamento aeróbio de 12 semanas em obesos e também observaram melhora nas atividades simpáticas e parassimpáticas do SNA.

Destaca-se ainda que o treinamento combinado utilizado se trata de um treinamento 
periodizado, com cargas individualizadas e submáximas e máximas. Uusitalo et al. $^{18}$ mostraram que o excesso de treinamento, chamado de "overtraining", está associado com alterações negativas nos marcadores de VFC, como a redução da atividade parassimpática. Desta forma, o monitoramento de cargas de treino é de suma importância para acompanhamento da evolução de atletas principalmente no que se refere a possíveis intervenções para ganho performance funcional e prevenção e lesões. Gisselman et $a .^{19}$ observaram relação entre a VFC e índices de lesão, caracterizando essa variável autonômica como uma importante ferramenta de monitoramento. Sendo assim, interpretar alterações relacionadas à VFC parece pertinente no âmbito esportivo.

No que se refere à relação da modulação autonômica cardíaca pela VFC e a performance funcional, Merati et al. $^{20}$ observaram uma correlação entre essas variáveis para atletas de elite de natação, mostrando que quanto maior o índice vagal de repouso maiores eram os tempos de tiros de $50 \mathrm{~m}$. Por outro lado, o índice de balanço simpático/vagal se correlacionou com bons resultados de performance funcional. No presente estudo este índice de balanço foi representado pelo índice SDNN, e observou-se melhora no mesmo após o programa de treinamento. Ao considerar o exposto, espera-se resultados positivos tanto para modulação autonômica cardíaca como de performance nos participantes que realizaram tal protocolo de treinamento.

Um ponto chave do presente estudo foi o treinamento proposto, tendo em vista seus benefícios sobre o desempenho funcional já citados $^{4}$. Sugere-se, portanto, futuros estudos que associem alterações na modulação autonômica cardíaca com ganhos funcionais visando explicar fenômenos característico do treinamento combinado.

\section{CONCLUSÃO}

A partir dos achados do presente estudo pode-se concluir que um treinamento combinado de sprints e agachamento durante seis semanas é eficaz na melhora dos índices do domínio do tempo da variabilidade da frequência cardíaca.

\section{CONFLITOS DE INTERESSE}

Os autores declaram não haver qualquer potencial conflito de interesses que possa interferir na imparcialidade deste trabalho científico.

\section{REFERÊNCIAS}

1. Barbanti VJ, Tricoli V, Ugrinowitsch C. Relevância do conhecimento científico na prática do treinamento físico. Rev Paul Educ Fís. 2004;(18):101-9.

2. Hartmann $H$, Wirth $K$, Keiner M, Mickel C, Sander A, Szilvas E. Short-term periodization models: effects on strength and speed-strength performance. Sports Med. 2015;45(10):1373-86. DOI: https://doi.org/10.1007/s40279-015-0355-2

3. Irving BA, Lanza IR, Henderson GC, Rao RR, Spiegelman BM, Nair KS. Combined training enhances skeletal muscle mitochondrial oxidative capacity independent of age. J Clin Endocrinol Metab. 2015;100(4):1654-63. DOI: https://doi.org/10.1210/ic.2014-3081

4. Marques M, Gabbett $T$, Marinho D, Blazevich $A$, Sousa A, van den Tillaar $R$ et al. Influence of strength, sprint running, and combined strength and sprint running training on short sprint performance in young adults. Int J Sports Med. 2015;36(10):789-95.

DOI:

\section{https://doi.org/10.1055/s-0035-1547284}

5. Eklund D, Pulverenti T, Bankers S, Avela J, Newton $R$, Schumann $M$ et al. Neuromuscular adaptations to different modes of combined strength and endurance training. Int J Sports Med. 2014;36(2):120-9. DOI: https://doi.org/10.1055/s-0034-1385883

6. Noon MR, James RS, Clarke ND, Akubat I, Thake CD. Perceptions of well-being and physical performance in English elite youth footballers across a season. J Sports Sci. 2015;33(20):210615.

DOI:

https://doi.org/10.1080/02640414.2015.1081393

7. Baar K. Training for endurance and strength: lessons from cell signaling. Med Sci Sports Exerc. 2006;38(11):1939-44.

8. Vanderlei LCM, Pastre CM, Hoshi RA, Carvalho TD, Godoy MF. Noções básicas de variabilidade 
da frequência cardíaca e sua aplicabilidade clínica. Rev Bras Cir Cardiovasc. 2009;24(2):20517. DOI: https://doi.org/10.1590/S0102$\underline{76382009000200018}$

9. Aubert $A E$, Seps B, Beckers F. Heart rate variability in athletes. Sports Med. 2003;33(12):889-919.

DOI: https://doi.org/10.2165/00007256-20033312000003

10. Pumprla J, Howorka K, Groves D, Chester M, Nolan J. Functional assessment of heart rate variability: physiological basis and practical applications. Int J Cardiol. 2002;84(1):1-14. DOI: https://doi.org/10.1016/S0167-5273(02)00057-8

11. Heart rate variability: Standards of measurement, physiological interpretation, and clinical use. Eur Heart J. 1996;17:28. DOI: https://doi.org/10.1093/eurhearti/17.suppl 2.28

12. Bellenger CR, Fuller JT, Thomson RL, Davison $\mathrm{K}$, Robertson EY, Buckley JD. Monitoring athletic training status through autonomic heart rate regulation: a systematic review and metaanalysis. Sports Med. 2016;46(10):1461-86. DOI: https://doi.org/10.1007/s40279-016-0484-2

13. Ascensão A, Leite M, Rebelo AN, Magalhäes S, Magalhäes J. Effects of cold water immersion on the recovery of physical performance and muscle damage following a one-off soccer match. J Sports Sci. 2011;29(3):217-25. DOI: https://doi.org/10.1080/02640414.2010.526132

14. Machado AF, Micheletti JK, Vanderlei FM, Nakamura FY, Leal-Junior ECP, Netto Junior J et al. Effect of low-level laser therapy (LLLT) and light-emitting diodes (LEDT) applied during combined training on performance and postexercise recovery: protocol for a randomized placebo-controlled trial. Braz J Phys Ther. 2017;21(4):296-304.

DOI: https://doi.org/10.1016/j.bjpt.2017.05.010

15. Marães VFSR, Teixeira LCA, Catai AM, Milan LA, Rojas FAR, Oliveira L et al. Determinação e avaliação do limiar de anaerobiose a partir de métodos de análise da frequência cardíaca de sua variabilidade. Rev SOCESP. 2003;13(4):1-6.

16. Vanin AA, Miranda EF, Machado CS, de Paiva $\mathrm{PR}$, Albuquerque-Pontes GM, Casalechi $\mathrm{HL}$ et al. What is the best moment to apply phototherapy when associated to a strength training program? A randomized, double-blinded, placebocontrolled trial : Phototherapy in association to strength training. Lasers Med Sci. 2016;31(8):1555-64. DOI: https://doi.org/10.1007/s10103-016-2015-7

Amano $\mathrm{M}$, Kanda $\mathrm{T}$, Ue $\mathrm{H}$, Moritani T. Exercise training and autonomic nervous system activity in obese individuals: Med Sci Sports Exerc. 2001;33(8):1287-91. DOI: https://doi.org/10.1097/00005768-200108000$\underline{00007}$

18. Uusitalo ALT, Uusitalo AJ, Rusko HK. Heart rate and blood pressure variability during heavy training and overtraining in the female athlete. 2000;21(1):45-53.

19. Gisselman AS, Baxter GD, Wright A, Hegedus $E$, Tumilty $S$. Musculoskeletal overuse injuries and heart rate variability: is there a link? Med Hypotheses. 2016;87:1-7. DOI: https://doi.org/10.1016/i.mehy.2015.12.003

20. Merati G, Maggioni MA, Invernizzi PL, Ciapparelli C, Agnello L, Veicsteinas A et al. Autonomic modulations of heart rate variability and performances in short-distance elite swimmers. Eur J Appl Physiol. 2015;115(4):82535. DOI: https://doi.org/10.1007/s00421-0143064-x

Recebido para publicação em 10/08/2018

Revisado em 25/08/2018

Aceito em 28/08/2018 